

А. Г. Шоломицкий, О необходимых условиях нормальной сходимости для мартингалов, Теория вероятн. и ее примен., 1998, том 43, выпуск 3, 490-508

DOI: https://doi.org/10.4213/tvp1556

Использование Общероссийского математического портала Math-Net.Ru подразумевает, что вы прочитали и согласны с пользовательским соглашением http://www . mathnet.ru/rus/agreement

Параметры загрузки:

IP: 54.80 .97 .219

26 апреля 2023 г., $16: 58: 10$

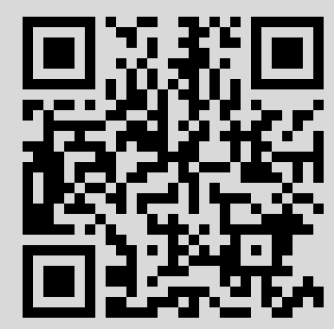


(c) $1998 \mathrm{r}$.

шоломицкий А. Г.

\section{О НЕОБХОДИМЫХ УСЛОВИЯХ НОРМАЛЬНОЙ СХОДИМОСТИ ДЛЯ МАРТИНГАЛОВ ${ }^{1)}$}

Работа посвящена доказательству необходимости выполнения условия Линдеберга для нормальной сходимости мартингалов в схеме серий при некоторых дополнительных условиях симметричности слагаемых. Выполнение «принципа инвариантности» не предполагается.

Ключевые слова и Фразы: «условное» условие Линдеберга, предельная пренебрегаемость, условные моменты.

1. Введение. Обобщения теоремы Линдеберга для мартингалов с использованием условных математических ожиданий и сходимости по вероятности предлагались в ряде работ (см., например, [6], [22] и библиографию там). Настоящая работа посвящена доказательству (при некоторых дополнительных условиях) утверждения, обратного следующей известной теореме, содержащейся, в частности, в результатах работ Брауна и Иглсона [1], Брауна [2] и Дворецкого [5].

Пусть при каждом $n$ на некотором вероятностном пространстве $(\Omega, \mathscr{F}, \mathbf{P})$ заданы случайные өеличины $\left\{X_{j, n}\right\}, 1 \leqslant j \leqslant k_{n}<\infty$.

Пусть $\mathscr{F}_{n}^{j-1}=\sigma\left(X_{1, n}, \ldots, X_{j-1, n}\right), \mathscr{F}_{n}^{0}=(\varnothing, \Omega) u$

$$
\mathrm{E}\left(X_{j, n} \mid \mathscr{F}_{n}^{j-1}\right)=0 \quad \text { (P-n.H.). }
$$

Предположим, что случаймые величины $X_{j, n}$ квадратично интегрируемы $u$

$$
\sum_{j=1}^{k_{n}} \mathbf{E}\left(X_{j, n}^{2} \mid \mathscr{F}_{n}^{j-1}\right) \stackrel{\mathrm{p}}{\longrightarrow} \sigma^{2}>0 .
$$

Тогда из выполнения «условного» условия Лиядеберга:

$$
\Lambda_{n}(\varepsilon)=\sum_{j=1}^{k_{n}} \mathbf{E}\left\{X_{j, n}^{2} I\left(\left|X_{j, n}\right| \geqslant \varepsilon\right) \mid \mathscr{F}_{n}^{j-1}\right\} \stackrel{\mathrm{p}}{\longrightarrow} 0
$$

*Центральный экономико-математический институт РАН, Нахимовский просп., 47, 117418 Москва, Россия.

1) Работа выполнена при поддержке Российского фонда фундаментальных исследований, грант 96-01-01229. 
при $n \rightarrow \infty$ для любого $\varepsilon>0$, следует, ито ${ }^{2)}$

$$
\mathscr{L}\left(\sum_{j} X_{j, n}\right) \longrightarrow \mathscr{N}\left(0, \sigma^{2}\right) \quad n p u n \rightarrow \infty
$$

(здесь и далее символом $I[\cdot]$ обозначается индикатор события).

В [1] содержится обобщение этого результата на случай предельного безгранично делимого закона с конечной дисперсией. В [5] показано, что теорема верна с заменой в ее формулировке $\sigma$-алгебр $\mathscr{F}_{n}^{j-1}$ на $\sigma$-алгебры, порожденные суммами $S_{n}(j-1)=X_{1, n}+\cdots+X_{j-1, n}$ (см. лемму 3.1 в [5]).

Приведенную выше теорему можно рассматривать как следствие доказанных поозднее общих теорем о функциональной сходимости мартингалов к гауссовским процессам. Центральной предельной теореме для мартингалов, в том числе в схеме серий, было посвящено большое количество работ (см., например, [21], [14], [19]). Наиболее общие результаты в этой области были получены Р. ІІ. Липцером и А. Н. Ширяевым [10]--[12] (см. также [6]).

Необходимые условия в функциональных теоремах о сходимости к непрерывному гауссовскому процессу были получены Ганслером и Хауслером [4], Ротзеном [17] для схемы серий, для общего случая Р. Ш. Липцером и А. Н. Ширяевым [11] (см. также [6], [16]). В указанных работах доказана, необходимость условия Линдеберга для функциональной сходимости гроцесса, построенного по значениям частичных сумм $S_{n}(1), \ldots, S_{n}\left(k_{n}\right)$, к гауссовскому процессу. Отличие результатов настоящей работы состоит в том, что вместо требования функциональной сходимости используется лишь условие сходимости сумм $(\mathrm{N})$. B следуюшем пункте будет приведен пример, поясняющий это различие.

В упомянутых выше работах [10]-[12] (см. также [7]) были развиты методы, позволяющие получать широкий класс предельных теорем для мартингалов. На эти методы (для дискретного случая) существенно опирается и настоящая работа.

Я хотел бы выразить свою благодарность В. И. Ротарю за постоянную помошь и большое внимание, проявленное к работе, а также В. М. Круглову за полезные замечания. Я благодарен рецензенту за замечания, способствовавшие улучшению работы.

2. Формулировка результатов. Пусть

$$
\max _{j \leqslant k_{n}} \mathbf{E}\left(X_{j, n}^{2} \mid \mathscr{F}_{n}^{j-1}\right) \stackrel{\mathrm{p}}{\longrightarrow} 0 \quad \text { при } n \rightarrow \infty .
$$

2) Ниже, как обычно, символом $\mathscr{L}(\xi)$ обозначается закон распределения случайной величины (с.в.) $\xi$, символом $\mathscr{L}\left(\xi_{n}\right) \rightarrow \mathscr{L}(\xi)$ - сходимость законов в смысле слабой сходимости соответствующих распределений. Нормальный захон с математичесхим ожиданием $m$ и дисперсией $\sigma^{2}$ обозначается через $\mathcal{N}\left(m, \sigma^{2}\right)$. 
Это условие предельной пренебрегаемости следует из (L).

Ниже приводятся утверждения о необходимости условия Линдеберга для (N) при выполнении (1)-(3) и дополнительных условиях «симметричности», которые могут вводиться в различной форме.

Теорема 1. Пусть выполнены условия (2) и (3). Если при $n \rightarrow \infty$

$$
\mathscr{L}\left(\sum_{j=1}^{k_{n}} c_{j, n} X_{j, n}\right) \longrightarrow \mathcal{N}\left(0, \sigma^{2}\right)
$$

для любых наборов чисел $c_{j, n}$, принимающих значения \pm 1, то выполнено условие (L).

Теорема будет доказана в п. 3.

3 а м е ч а н и е. Условие $\left(\mathrm{N}_{\mathrm{c}}\right)$ следует из $(\mathrm{N})$ в том случае, когда условные распределения с.в. $X_{j, n}$ относительно $\sigma$-алгебр $\mathscr{F}_{n}^{j-1} \mathbf{P}$-п.н. симметричны. Поэтому сформулированная теорема дает необходимые условия нормальной сходимости для такого «симметричного» случая (см. также утверждение А в п. 3). В общем случае условие $\left(\mathrm{N}_{\mathrm{c}}\right)$ позволяет при доказательстве теоремы произвести определенного рода симметризацию слагаемых (см. доказательство теоремы в п. 3). Этим, в частности, объясняется то, что в теореме формально не нужно условие мартингальности (1).

Требование симметричности можно ослабить. Так, если наложить моментное ограничение

$$
\sup _{n} \sum_{j=1}^{k_{n}} \mathbf{E}\left|X_{j, n}\right|^{5} \leqslant M_{0}<\infty,
$$

то в качестве условия «симметричности» можно использовать условие

$$
\sum_{j<i} \mathbf{E} X_{i, n}^{3} X_{j, n}=0 .
$$

Заметим, что условие (4) достаточно слабо, поскольку оно означает требование ограниченности суммы моментов «уже отнормированных» с.в., т.е. таких, для которых верно (2).

Теорема 2. Пусть выполнены условия (1)-(5). Тогда из (N) слеdyem $(\mathrm{L})$.

Доказательство теоремы 2 приведено в п. 4.

Возможно, более удобная форма условий дается следующим следствием из теоремы 2. Рассмотрим условие

$$
\sum_{j<i} \mathbf{E} X_{i, n}^{3} X_{j, n} I\left(\left|X_{i, n}\right| \leqslant 1\right) I\left(\left|X_{j, n}\right| \leqslant 1\right) \longrightarrow 0 .
$$

Следствие. Пусть выполнены условия (1)-(3) $u$ (5'). Пусть $\mathbf{E} \Lambda(1) \longrightarrow 0$ nрu $n \rightarrow \infty$. Тогда из (N) следует (L). 
Доказательство следствия приведено в п. 5.

Как отмечалось выше, в условиях сформулированных теорем распределения частичных сумм могут не быть асимптотически нормальными (или даже взвешенными по дисперсии нормальными) и, соответственно, «принцип инвариантности» может не иметь места. Рассмотрим один пример такой ситуации, частично заимствованный из [5].

ПІ р и м е р. Пусть $k_{n}=3 n$. Пусть $\xi_{j, n}, j \leqslant 3 n$, независимы и $\mathscr{L}\left(\xi_{j, n}\right)=\mathscr{N}(0,1 / n)$. Положим

$$
\begin{aligned}
& X_{j, n}=\xi_{j, n} \quad \text { при } j \leqslant n ; \quad X_{j, n}=\xi_{j, n} I\left[S_{n}(n)>0\right] \quad \text { при } n<j \leqslant 2 n ; \\
& X_{j, n}=\xi_{j, n} I\left[S_{n}(n) \leqslant 0\right] \quad \text { при } 2 n<j \leqslant 3 n .
\end{aligned}
$$

Тогда выполнены условия $(\mathrm{L}),\left(\mathrm{N}_{\mathrm{c}}\right)$ и $(1)-(3)$ с $\sigma^{2}=2$. Кроме того, условные распределения с.в. $X_{j, n}$ относительно $\sigma$-алгебр $\mathscr{F}_{n}^{j-1} \mathbf{P}$-п.н. симметричны. Однако, например, для $m_{n} \leqslant 2 n$ суммы условных дисперсий первых $m_{n}$ слагаемых равны $1+I\left[S_{n}(n)>0\right] m_{n} / n$, и частичные суммы

$$
S_{n}\left(m_{n}\right)=S_{n}(n)+\left\{\xi_{n+1, n}+\cdots+\xi_{m_{n}, n}\right\} I\left[S_{n}(n)>0\right],
$$

как легко видеть, не являются асимптотически нормальными. Например, для $m_{n}=2 n$

$$
\mathscr{L}\left(S_{n}(2 n)\right)=\mathscr{L}\left(\xi_{1}+\xi_{2} I\left[\xi_{1}>0\right]\right),
$$

где $\xi_{1}$ и $\xi_{2}-$ независимые стандартно нормальные с.в. Как нетрудно проверить,

$$
\mathbf{P}\left\{S_{n}(2 n)>0\right\}=\frac{1}{2} \mathbf{P}\left\{\xi_{1}+\xi_{2}>0 \mid \xi_{1}>0\right\}<\frac{1}{2},
$$

т.е. распределение с.в. $S_{n}(2 n)$ не только не является взвесью нормальных по дисперсии, но даже не симметрично.

3. Доказательство теоремы 1. Для удобства, не ограничивая общности, положим $\sigma^{2}=1$. Кроме того, договоримся наделять всю последовательность индексов $n$ свойствами, верными для ее подпоследовательностей в тех случаях, когда это не повлечет недоразумений.

Доказательство теоремы опирается на формулируемое ниже утверждение.

Утверждение А. Пусть при каждом $n\left\{Y_{j, n}, \mathscr{K}_{n}^{j}\right\}, 1 \leqslant j \leqslant k_{n},-$ стохастическая последовательность такая, ито для каждой из случайных величин. $Y_{j, n}$ ее условные распределения относительно б-алгебры $\mathscr{K}_{n}^{j-1}$ P-n.н. симметричны. Пусть

$$
\mathscr{L}\left(\sum_{j=1}^{k_{n}} Y_{j, n}\right) \longrightarrow \mathcal{N}(0,1) \quad \text { npu } n \rightarrow \infty
$$


и выполнены условия:

$$
\sum_{j=1}^{k_{n}} \mathbf{E}\left\{Y_{j, n}^{2} \mid \mathscr{K}_{n}^{j-1}\right\} \stackrel{\mathrm{p}}{\longrightarrow} 1 \quad \text { npu } n \rightarrow \infty
$$

существует такая последовательность положительных чисел $\lambda_{n} \rightarrow 0$, чmo

$$
\max _{j \leqslant k_{n}} \mathbf{E}\left\{Y_{j, n}^{2} \mid \mathscr{K}_{n}^{j-1}\right\} \leqslant \lambda_{n} \quad \text { (P-n.H.) }
$$

для некоторого числа $M_{1}<\infty$

$$
\sum_{j=1}^{k_{n}} \mathbf{E}\left\{Y_{j, n}^{2} \mid \mathscr{K}_{n}^{j-1}\right\} \leqslant M_{1}
$$

Тогда выполнено условие Линдеберга: для любого $\varepsilon>0$

$$
\sum_{j=1}^{k_{n}} \mathbf{E}\left\{Y_{j, n}^{2} I\left(\left|Y_{j, n}\right| \geqslant \varepsilon\right)\right\} \longrightarrow 0 \quad \text { npu } n \rightarrow \infty .
$$

Доказательст во утверждения А. Положим

$$
\beta_{j, n}^{2}=\mathbf{E}\left\{Y_{j, n}^{2} \mid \mathscr{K}_{n}^{j-1}\right\}, \quad Y_{n}=\sum_{j=1}^{k_{n}} Y_{j, n} .
$$

Пусть $f_{j, n}(t)=\mathbf{E}\left\{\exp \left(i t Y_{j, n}\right) \mid \mathscr{K}_{n}^{j-1}\right\}-$ условные характеристические функции (у.х.ф.) с.в. $Y_{j, n}, F_{j, n}\left(d x \mid \mathscr{K}_{n}^{j-1}\right)-$ соответствуюшие условные распределения. По условию существуют такие регулярные варианты условных распределений, что $F_{j, n}\left(d x \mid \mathscr{K}_{n}^{j-1}\right)$ при всех $\omega \in \Omega$ является симметричной мерой и условия (7) и (8) выполнены. Этот вариант каждого из условных распределений и условимся рассматривать ниже.

Обозначим

$$
\mathscr{E}_{n}(t)=\prod_{j} f_{j, n}(t), \quad \mathscr{J}_{n}(t)=\exp \left\{\int_{-\infty}^{+\infty}\left(e^{i t x}-1-i t x\right) x^{-2} d K_{n}(x)\right\}
$$

где $K_{n}(u)=\sum_{j=1}^{k_{n}} \int_{-\infty}^{u} x^{2} F_{j, n}\left(d x \mid \mathscr{K}_{n}^{j-1}\right)$ - случайная функция, имеющая в силу (8) ограниченную вариацию. Пусть $K_{n}(d x)$ - соответствующая функции $K_{n}(u)$ (случайная) мера.

Поскольку распределения $F_{j, n}\left(d x \mid \mathscr{K}_{n}^{j-1}\right)$ симметричны, у.х.ф. $f_{j, n}(t)$ вешественны и мера $K_{n}$ симметрична, поэтому

$$
\begin{aligned}
\mathscr{J}_{n}(t) & =\exp \left\{\int_{-\infty}^{+\infty}\left(e^{i t x}-1-i t x\right) x^{-2} d K_{n}(x)\right\} \\
& =\exp \left\{\int_{-\infty}^{+\infty}(\cos (t x)-1) x^{-2} d K_{n}(x)\right\} .
\end{aligned}
$$


Из (8) получаем оценку

$$
\left|\ln \mathscr{J}_{n}(t)\right|=\left|\int_{-\infty}^{+\infty}(\cos (t x)-1) x^{-2} K_{n}(d x)\right| \leqslant \frac{t^{2}}{2} \operatorname{Var} K_{n} \leqslant M_{1} \frac{t^{2}}{2}
$$

где $\operatorname{Var} K_{n}=K(+\infty)-K(-\infty)=K(+\infty)$.

Зафиксируем произвольное $t$. Так как, согласно (7),

$$
\begin{aligned}
\max _{j}\left|f_{j, n}(t)-1\right| & =\max _{j}\left|f_{j, n}(t)-1-\mathbf{E}\left\{Y_{j, n} \mid \mathscr{K}_{n}^{j-1}\right\}\right| \\
& \leqslant \frac{t^{2}}{2} \max _{j} \beta_{j, n}^{2} \leqslant \frac{\lambda_{n} t^{2}}{2} \longrightarrow 0 \text { при } n \rightarrow \infty,
\end{aligned}
$$

можно указать такое $n_{t}$, что $f_{j, n}(t) \geqslant \frac{1}{2}$ при всех $j$ для $n \geqslant n_{t}$. Следовательно, $\ln f_{j, n}(t)$ при $n \geqslant n_{t}$ сушествует и конечен. Тогда из (7) и (8) имеем

$$
\begin{aligned}
& \left|\ln \mathscr{E}_{n}(t)-\sum_{j=1}^{k_{n}}\left(f_{j, n}(t)-1\right)\right|=\left|\ln \prod_{j} f_{j, n}(t)-\sum_{j=1}^{k_{n}}\left(f_{j, n}(t)-1\right)\right| \\
& \quad \leqslant \sum_{j=1}^{k_{n}}\left|f_{j, n}(t)-1\right|^{2}=\sum_{j=1}^{k_{n}}\left|f_{j, n}(t)-1-\mathbf{E}\left(Y_{j, n} \mid K_{n}^{j-1}\right)\right|^{2} \\
& \quad \leqslant \frac{t^{4}}{4} \sum_{j=1}^{k_{n}} \beta_{j, n}^{4} \leqslant \frac{t^{4}}{4} \max _{j} \beta_{j, n}^{2} \sum_{j=1}^{k_{n}} \beta_{j, n}^{2} \leqslant \frac{t^{4}}{4} \lambda_{n} M_{1} \longrightarrow 0 \text { при } n \rightarrow \infty .
\end{aligned}
$$

Кроме того, из (8)

$$
\left|\sum_{j=1}^{k_{n}}\left(f_{j, n}(t)-1\right)\right|=\left|\sum_{j=1}^{k_{n}}\left(f_{j, n}(t)-1-\mathbf{E}\left(Y_{j, n} \mid K_{n}^{j-1}\right)\right)\right| \leqslant \frac{t^{2}}{2} M_{1} .
$$

Поскольку величины $\mathscr{J}(t)$ и $\mathscr{E}(t)$ вешественны, из сделанных оценок следует существование при каждом $t$ таких констант $c_{1}(t)<\infty$ и $c_{2}(t)<\infty$, что для всех $n$, больших некоторого номера (зависяцего от $t$ ),

$$
\left|\mathscr{J}_{n}(t)\right|^{-1} \leqslant c_{1}(t), \quad\left|\mathscr{E}_{n}(t)\right|^{-1} \leqslant c_{2}(t) .
$$

Кроме того, поскольку $\ln \mathscr{J}_{n}(t)=\sum_{j}\left(f_{j, n}(t)-1\right)$ и $\left|\mathscr{J}_{n}(t)\right| \leqslant 1$, пользуясь неравенством $\left|e^{x}-1\right| \leqslant|x| e^{|x|}$, имеем

$$
\begin{aligned}
\mathbf{E}\left|\mathscr{E}_{n}(t)-\mathscr{J}_{n}(t)\right| & \leqslant \mathbf{E}\left|\exp \left[\ln \mathscr{E}_{n}(t)-\ln \mathscr{J}_{n}(t)\right]-1\right| \\
& \leqslant \exp \left(t^{2} M_{1}\right) \mathbf{E}\left|\ln \mathscr{E}_{n}(t)-\ln \mathscr{J}_{n}(t)\right| \\
& \leqslant \exp \left(t^{2} M_{1}\right) \frac{t^{4}}{4} \lambda_{n} M_{1} \longrightarrow 0 \text { при } n \rightarrow \infty
\end{aligned}
$$


Теперь, используя сделанные оценки, получаем для всех $n$, больших некоторого зависящего от $t$ номера:

$$
\begin{aligned}
& \left|\mathbf{E} \mathscr{J}_{n}^{-1}(t) \exp \left(i t Y_{n}\right)-\mathbf{E} \mathscr{E}_{n}^{-1}(t) \exp \left(i t Y_{n}\right)\right| \\
& \quad=\left|\mathbf{E}\left[\mathscr{E}_{n}^{-1}(t)-\mathscr{J}_{n}^{-1}(t)\right] \exp \left(i t Y_{n}\right)\right| \\
& \quad \leqslant c_{1}(t) c_{2}(t) \mathbf{E}\left|\mathscr{J}_{n}(t)-\mathscr{E}_{n}(t)\right| .
\end{aligned}
$$

В силу (9) последнее выражение стремится к нулю при $n \rightarrow \infty$.

Поскольку при любом фиксированном $t$ и всех $n$ таких, что $f_{j, n}(t)>0$ для всех $j$ (см. [10]-[12], [22, с. 58]),

$$
\mathbf{E} \mathscr{E}_{n}^{-1}(t) \exp \left(i t Y_{n}\right)=\mathbf{E} \prod_{j} \frac{e^{i t X_{j, n}}}{f_{j, n}(t)}=1,
$$

отсюда

$$
\mathbf{E} \mathscr{J}_{n}^{-1}(t) \exp \left(i t Y_{n}\right) \longrightarrow 1 \quad \text { при } n \rightarrow \infty .
$$

Далее, положим

$$
\begin{aligned}
\psi_{n}(t) & =i t Y_{n}-\int_{-\infty}^{+\infty}\left(e^{i t x}-1-i t x\right) x^{-2} K_{n}(d x) \\
& =i t Y_{n}-\int_{-\infty}^{+\infty}(\cos (t x)-1) x^{-2} K_{n}(d x) .
\end{aligned}
$$

Совокупность с.в. $\left\{\psi_{n}(t)\right\}_{t=-\infty}^{+\infty}$ является случайным процессом (с.п.) с непрерывными траекториями. Нетрудно показать (см. [3], [6] или [22, c. 192-193]), что $\psi_{n}(\cdot)$ можно рассматривать как случайный элемент, принимающий значения в пространстве $\left(C_{\mathrm{lu}}, \mathscr{B}\right)$ непрерывных функщий из $\mathbf{R}^{1}$ в $\mathbf{C}$ с локально равномерной топологией, задаваемой метрикой

$$
\delta_{\mathrm{lu}}(f, g)=\sum_{N=1}^{\infty} 2^{-N}\left(1,|f-g|_{N}\right)
$$

где $|f|_{N}=\sup _{|t| \leqslant N}|f(t)|$, и соответствующей этой топологии борелевской $\sigma$-алгеброй $\mathscr{B}$.

Используя известные критерии компактности множеств распределений в метрических пространствах (см. [3], [6], [9], [15], [20]), можно показать, что последовательность распределений случайных элементов $\psi_{n}(\cdot)$ относительно слабо компактна в $\left(C_{\mathrm{lu}}, \mathscr{B}\right)$. Это устанавливается, например, с помощью предложений 3.26 и $1.17[6$, гл. VI].

Выделим из последовательности распределений случайных элементов $\psi_{n}(\cdot)$ подпоследовательность, слабо сходящуюся к распределению некоторого случайного элемента $\psi(\cdot)$ со значениями в $\left(C_{\mathrm{lu}}, \mathscr{B}\right)$. Так как пространство $C_{\text {lu }}$ является польским (см. $[6$, с. 449]), согласно известной теореме А. В. Скорохода [18] (см. также $[22$, с. 380]), можно ввести 
новое вероятностное пространство $\Omega^{*}$ с мерой $\mathbf{P}^{*}$ и определить на нем случайные элементы $\psi_{n}^{*}\left(\cdot, \omega^{*}\right)$ и $\psi^{*}\left(\cdot, \omega^{*}\right)$ со значениями в $\left(C_{\mathrm{lu}}, \mathscr{B}\right)$ так, чтобы

$$
\begin{aligned}
& \psi_{n}^{*}\left(\cdot, \omega^{*}\right) \stackrel{\mathrm{d}}{=} \psi_{n}(\cdot), \quad \psi^{*}\left(\cdot, \omega^{*}\right) \stackrel{\mathrm{d}}{=} \psi(\cdot), \\
& \mathbf{P}^{*}\left\{\omega^{*} \in \Omega^{*}: \psi_{n}^{*}\left(\cdot, \omega^{*}\right) \stackrel{\mathrm{lu}}{\longrightarrow} \psi^{*}\left(\cdot, \omega^{*}\right)\right\}=1,
\end{aligned}
$$

где символом $\stackrel{\text { lu }}{\longrightarrow}$ обозначается сходимость в локально равномерной топологии, $\omega^{*}$ - элементарное событие пространства $\Omega^{*}$.

В свою очередь, $\left\{\psi_{n}^{*}(t)\right\}_{t=-\infty}^{+\infty}$ можно рассматривать как с.п. (т.е. набор с.в.) (см., например, [3], [6], [22, с. 192]). Для каждого полуинтервала $[a, b)$ согласно известной формуле обращения для преобразований Фурье мер (см., например, [13, с. 199]) положим

$$
K_{n}^{*}[a, b)=\lim _{U \rightarrow \infty}(2 \pi)^{-1} \int_{-U}^{+U}\left(e^{-i u a}-e^{i u b}\right)(i u)^{-1}\left(\psi_{n}^{*}\right)^{\prime \prime}(u) d u
$$

где $\left(\psi_{n}^{*}\right)^{\prime \prime}(u)$ - вторая производная указанной функции. Распределение $\psi_{n}^{*}\left(\cdot, \omega^{*}\right)$ сосредоточено на множестве таких $\omega^{*}$, что указанные величины существуют, конечны, неотрицательны и, так как $\psi_{n}^{*}$ действительна, $K_{n}^{*}$ - симметричная мера, то $K_{n}^{*}[a, b)=K_{n}^{*}(-b,-a]$; поэтому условимся рассматривать только такие $\omega^{*}$. Из определения $K_{n}^{*}[a, b)$ видно, что величина $K_{n}^{*}(A)$ для любого борелевского $A \subseteq \mathbf{R}^{1}$ представима в виде предела с.в., и, следовательно, сама является с.в. Далее, положим

$$
i Y_{n}^{*}=\psi_{n}^{*}\left(1, \omega^{*}\right)+\int_{-\infty}^{+\infty}\left(e^{i x}-1-i x\right) x^{-2} K_{n}^{*}(d x)
$$

Так определенная $Y_{n}^{*}$ тоже является с.в. как предел с.в. Так как $Y_{n}^{*}$ и $K_{n}^{*}(A)$ определяются однозначно по значениям $\psi_{n}^{*}(t)$ в рациональных точках и $\sigma$-алгебра $\mathscr{B}$ содержит цилиндрические множества, из того, что $\psi_{n}^{*}\left(\cdot, \omega^{*}\right) \stackrel{\mathrm{d}}{=} \psi_{n}(\cdot)$, следует, что $Y_{n}^{*} \stackrel{\mathrm{d}}{=} Y_{n}$ и $K_{n}^{*}(A) \stackrel{\mathrm{d}}{=} K_{n}(A)$.

Положим $K_{n}^{*}\left(x, \omega^{*}\right)=K_{n}^{*}(-\infty, x)$. Определенные описанным образом $Y_{n}^{*}=Y_{n}^{*}\left(\omega^{*}\right)$ и $K_{n}^{*}\left(x, \omega^{*}\right)$ удовлетворяют соотношению

$$
\psi_{n}^{*}\left(t, \omega^{*}\right)=i t Y_{n}^{*}\left(\omega^{*}\right)-\int_{-\infty}^{+\infty}(\cos (t x)-1) x^{-2} d K_{n}^{*}\left(x, \omega^{*}\right)
$$

Положим $B=\left\{\omega^{*} \in \Omega^{*}: \psi_{n}^{*}\left(\cdot, \omega^{*}\right) \stackrel{\text { lu }}{\longrightarrow} \psi^{*}\left(\cdot, \omega^{*}\right)\right\}$. Пользуясь известными фактами теории безгранично делимых характеристических функций (см., например, $[13, \S 21,22]$ ), нетрудно показать, что множество функций вида

$$
\phi(t)=i t \gamma-\int_{-\infty}^{+\infty}(\cos (t x)-1) x^{-2} d K(x)
$$


где $\gamma$ - число, $K$ - неубывающая функция, при условии равномерной ограниченности $\operatorname{Var} K$ замкнуто относительно сходимости в локально равномерной топологии. Кроме того, из сходимости последовательности функций $\phi_{n}(t)$ к функции $\phi(t)$ в локально равномерной топологии следует сходимость соответствуюших констант $\gamma_{n}$ и функций $K_{n}$ (во всех точках непрерывности) к предельным.

Следовательно, при каждом $\omega^{*} \in B$ существуют такие число $Y=$ $Y\left(\omega^{*}\right)$ и неубываюцая функция $\left\{K(u)=K\left(u, \omega^{*}\right)\right\}_{u=-\infty}^{+\infty}$, что для $\omega^{*} \in B$ верно представление

$$
\psi^{*}\left(t, \omega^{*}\right)=i t Y\left(\omega^{*}\right)-\int_{-\infty}^{+\infty}(\cos (t x)-1) x^{-2} d K\left(x, \omega^{*}\right)
$$

где $Y\left(\omega^{*}\right)=\lim _{n \rightarrow \infty} Y_{n}^{*}\left(\omega^{*}\right)$ и $K\left(u, \omega^{*}\right)=\lim _{n \rightarrow \infty} K_{n}^{*}\left(u, \omega^{*}\right)$ при каждом $u$ таком, что $K\left(u, \omega^{*}\right)$ непрерывна в точке $u$ при данном $\omega^{*} \in B$, а в прочих точках $K\left(u, \omega^{*}\right)$ определяется по непрерывности слева. Так как $\mathbf{P}^{*}(B)=1$, отсюда следует, что $Y\left(\omega^{*}\right)$ и $K\left(u, \omega^{*}\right)$ есть $\mathbf{P}^{*}$-п.н. пределы последовательностей с.в. и, следовательно, тоже являются с.в. (см., например, $\left[8\right.$, теорема $4^{\prime}$, гл. $\left.\mathrm{V}, \S 4\right)$. Меру, задаваемую приращениями функции $K$, обозначим той же буквой $K$. Нетрудно показать, что $K(A)$ для любого борелевского $A$ есть с.в. и что интеграл от любой измеримой функции по указанной мере также есть с.в.

Предельная с.в. $Y$ распределена стандартно нормально, так как

$$
\mathscr{L}\left(Y_{n}^{*}\right)=\mathscr{L}\left(Y_{n}\right) \longrightarrow \mathscr{N}(0,1) \text { при } n \rightarrow \infty .
$$

Так как, по доказанному $K_{n}(A) \stackrel{\mathrm{d}}{=} K_{n}^{*}(A)$ и, согласно $(6), K_{n}\left(\mathbf{R}^{1}\right) \stackrel{\mathrm{p}}{\longrightarrow} 1$, имеем $K_{n}^{*}\left(\mathbf{R}^{\mathbf{1}}\right) \stackrel{\mathrm{p}}{\longrightarrow} 1$. Поскольку вариация предельной меры не превосходит предела вариаций допредельных (см., например, $[13$, c. 191]), отсюда

$$
\operatorname{Var} K \leqslant 1 \quad \text { (P-п.н.). }
$$

При каждом фиксированном $t$ распределение величины $\exp \left[\psi_{n}^{*}\left(t, \omega^{*}\right)\right]$ сосредоточено на ограниченном интервале. По теореме Лебега о мажорируемой сходимости

$$
\mathbf{E} \exp \left[\psi_{n}^{*}\left(t, \omega^{*}\right)\right] \longrightarrow \mathbf{E} \exp \left[\psi^{*}\left(t, \omega^{*}\right)\right]
$$

откуда в силу (10) $\mathbf{E} \exp \left[\psi^{*}\left(t, \omega^{*}\right)\right]=1$, или

$$
\mathbf{E} \exp \left\{i t Y-\int_{-\infty}^{+\infty}(\cos (t x)-1) x^{-2} K(d x)\right\}=1 .
$$

Если продифференцировать два раза по $t$ под знаком математического ожидания левую часть этого равенства, получится абсолютно сходящийся интеграл, поэтому операции дифференцирования и интегрирования 
можно менять местами. Приравнивая вторые производные правой и левой частей в точке $t=0$, получаем $\mathbf{E} \operatorname{Var} K-\mathbf{E} Y^{2}=\mathbf{E} \operatorname{Var} K-1=0$, откуда в силу (11) $\operatorname{Var} K=1$ (Р-п.н.). Тогда (12) можно переписать в виде

$$
E \exp \left[i t Y-\int_{-\infty}^{+\infty}\left(\cos (t x)-1+\frac{(t x)^{2}}{2}\right) x^{-2} K(d x)\right]=\exp \left(-\frac{t^{2}}{2}\right)
$$

или, поскольку с.в. $Y$ стандартно нормальна,

$$
\mathrm{E} \exp (i t Y)\left[1-\exp \left\{-\int_{-\infty}^{+\infty}\left(\cos (t x)-1+\frac{(t x)^{2}}{2}\right) x^{-2} K(d x)\right\}\right]=0
$$

Взяв здесь вещественную часть и разделив ее на $t^{4}$, получаем для ненулевого $t$

$$
\begin{aligned}
0= & t^{-4} \mathbf{E} \cos (t Y)\left[1-\exp \left\{-\int_{-\infty}^{+\infty}\left(\cos (t x)-1+\frac{(t x)^{2}}{2}\right) x^{-2} K(d x)\right\}\right] \\
= & t^{-4} \mathbf{E} \cos (t Y) I\{|t Y| \geqslant 1\} \\
& \times\left[1-\exp \left\{-\int_{-\infty}^{+\infty}\left(\cos (t x)-1+\frac{(t x)^{2}}{2}\right) x^{-2} K(d x)\right\}\right] \\
& +t^{-4} \mathbf{E} \cos (t Y) I\{|t Y|<1\} \\
& \times\left[1-\exp \left\{-\int_{-\infty}^{+\infty}\left(\cos (t x)-1+\frac{(t x)^{2}}{2}\right) x^{-2} K(d x)\right\}\right]
\end{aligned}
$$

Оценим первый член в правой части (13). Так как $\cos (x)-1+x^{2} / 2 \geqslant$ 0, выражение в квадратных скобках положительно и не превосходит единицы. Поэтому

$$
\begin{aligned}
& \mid t^{-4} \mathbf{E} \cos (t Y) I\{|t Y| \geqslant 1\} \\
& \quad \times\left[1-\exp \left\{-\int_{-\infty}^{+\infty}\left(\cos (t x)-1+\frac{(t x)^{2}}{2}\right) x^{-2} K(d x)\right\}\right] \mid \\
& \quad \leqslant t^{4} \mathbf{P}\left\{|Y| \geqslant|t|^{-1}\right\} .
\end{aligned}
$$

Рассмотрим второй член в правой части (13). Пользуясь элементарными неравенствами:

$$
\int_{-\infty}^{+\infty}\left(\cos (t x)-1+\frac{(t x)^{2}}{2}\right) x^{-2} K(d x) \leqslant t^{2} \operatorname{Var} K
$$




$$
\begin{aligned}
& 1-e^{-x}>\frac{x}{e} \quad \text { для } x \in(0,1), \quad \cos 1>\frac{1}{2}, \\
& x^{-4}\left[\cos x-1+\frac{x^{2}}{2}\right]>\frac{\cos 1}{4 !}>\frac{1}{48} \quad \text { для }|x|<1,
\end{aligned}
$$

получим для любых $t$ и $\varepsilon$ таких, что $0<|t|<1$ и $0<\varepsilon<|t|^{-1}$ :

$$
\begin{array}{rl}
t^{-4} & \mathbf{E} \cos (t Y) I\{|t Y|<1\} \\
& \times\left\{1-\exp \left[-\int_{-\infty}^{+\infty}\left(\cos (t x)-1+\frac{(t x)^{2}}{2}\right) x^{-2} K(d x)\right]\right\} \\
\geqslant & (2 e)^{-1} \mathbf{E} I\{|t Y|<1\} \int_{-\infty}^{+\infty}\left(\cos (t x)-1+\frac{(t x)^{2}}{2}\right)(t x)^{-4} x^{2} K(d x) \\
\geqslant & (2 e)^{-1} \mathbf{E} I\{|t Y|<1\} \int_{|t x|<1}\left(\cos (t x)-1+\frac{(t x)^{2}}{2}\right)(t x)^{-4} x^{2} K(d x) \\
\geqslant & (96 e)^{-1} \mathbf{E} I\{|t Y|<1\} \int_{|x|<1 /|t|} x^{2} K(d x) \\
\geqslant & (96 e)^{-1} \mathbf{E} I\left\{|Y|<|t|^{-1}\right\} \int_{\varepsilon<|x|<1 /|t|} x^{2} K(d x) \\
\geqslant & \varepsilon^{2}(96 e)^{-1} \mathbf{E} I\left\{|Y|<|t|^{-1}\right\} K\left\{x: \varepsilon \leqslant|x|<|t|^{-1}\right\} .
\end{array}
$$

Окончательно, из (13) и (14) имеем для всех $0<|t|<1$ и $0<\varepsilon<1 /|t|$

$$
t^{-4} \mathbf{P}\left\{|Y| \geqslant|t|^{-1}\right\} \geqslant \varepsilon^{2}(96 e)^{-1} \mathbf{E} I\left\{|Y|<|t|^{-1}\right\} K\left\{x: \varepsilon \leqslant|x|<|t|^{-1}\right\} .
$$

Пусть $L>\varepsilon$. Тогда для всех $|t|<1 / L$

$$
t^{-4} \mathbf{P}\left\{|Y| \geqslant|t|^{-1}\right\} \geqslant \varepsilon^{2}(96 e)^{-1} \mathbf{E} I\left\{|Y|<|t|^{-1}\right\} K\{x: \epsilon \leqslant|x|<L\} .
$$

Перейдем в этом неравенстве к пределу при $t \rightarrow 0$. Так как с.в. $Y$ распределена нормально, $t^{-4} \mathbf{P}\{|Y| \geqslant 1 /|t|\} \rightarrow 0$ при $t \rightarrow 0$. По теореме о монотонной сходимости

$$
\mathbf{E} I\left\{|Y|<|t|^{-1}\right\} K\{x: \varepsilon \leqslant|x|<L\} \rightarrow \mathbf{E} K\{x: \varepsilon \leqslant|x|<L\} \quad \text { при } t \rightarrow 0,
$$

и окончательно получаем $0 \geqslant \mathbf{E} K\{x: \varepsilon \leqslant|x| \leqslant L\}$, т.e. $\mathbf{E} K\{x: \varepsilon \leqslant|x| \leqslant$ $L\}=0$ для любых положительных $\varepsilon$ и $L$ таких, что $\varepsilon<L$.

Отсюда, как нетрудно показать, следует, что случайная мера $K$ п.н. сосредоточена в нуле с весом 1 . Поэтому множество $(-\infty,-\varepsilon] \cup[\varepsilon,+\infty)$ для любого $\varepsilon>0$ является множеством непрерывности меры $K$. Тогда при $n \rightarrow \infty$ п.н.

$$
K_{n}^{*}(-\infty,-\varepsilon]+K_{n}^{*}[\varepsilon,+\infty) \longrightarrow K(-\infty,-\varepsilon]+K[\varepsilon,+\infty)=0,
$$

откуда по теореме Лебега $\mathbf{E}\left\{K_{n}^{*}(-\infty,-\varepsilon]+K_{n}^{*}[\varepsilon,+\infty)\right\} \longrightarrow 0$. 
Так как, согласно сказанному выше, $K_{n}(-\infty,-\varepsilon]+K_{n}[\varepsilon,+\infty) \stackrel{\mathrm{d}}{=}$ $K_{n}^{*}(-\infty,-\varepsilon]+K_{n}^{*}[\varepsilon,+\infty)$, отсюда

$$
\begin{aligned}
\sum_{j=1}^{k_{n}} \mathbf{E}\left\{Y_{j, n}^{2} I\left(\left|Y_{j, n}\right| \geqslant \varepsilon\right)\right\} & =\mathbf{E}\left\{K_{n}(-\infty,-\varepsilon]+K_{n}[\varepsilon,+\infty)\right\} \\
& =\mathbf{E}\left\{K_{n}^{*}(-\infty,-\varepsilon]+K_{n}^{*}[\varepsilon,+\infty)\right\} \longrightarrow 0
\end{aligned}
$$

т.е. верно $\left(L^{\prime}\right)$.

Вообще говоря, $\left(\mathrm{L}^{\prime}\right)$ доказано для некоторой подпоследовательности значений $n$. Поскольку, как видно из доказательства, такую подпоследовательность можно выделить из любой подпоследовательности значений $n,\left(\mathrm{~L}^{\prime}\right)$ доказано.

Доказате льст в о т е о ре мы 1 . Положим $\delta_{j, n}^{2}=\mathbf{E}\left(X_{j, n}^{2} \mid\right.$ $\left.\mathscr{F}_{n}^{j-1}\right)$. Пусть для с.в. $\left\{X_{j, n}\right\}$ выполнены условия теоремы. Введем с.в., $\theta_{j, n}$, независимые при каждом $n$ от всех с.в. $\left\{X_{j, n}\right\}$ и между собою, каждая из которых принимает значения 1 и -1 с вероятностями $\frac{1}{2}$. Положим $Z_{j, n}=\theta_{j, n} X_{j, n}$. Пусть $\mathscr{K}_{n}^{k}=\sigma\left(\theta_{1, n}, \ldots, \theta_{k, n}, X_{1, n}, \ldots, X_{k, n}\right), \mathscr{K}_{n}^{0}-$ тривиальная $\sigma$-алгебра. Условные распределения с.в. $Z_{j, n}$ при условии $\mathscr{K}_{n}^{j-1}$ Р-п.н. симметричны. Кроме того, $\left|Z_{j, n}\right|=\left|X_{j, n}\right|$ и

$$
\mathbf{E}\left[Z_{j, n}^{2} \mid \mathscr{K}_{n}^{j-1}\right]=\mathbf{E}\left[X_{j, n}^{2} \mid \mathscr{K}_{n}^{j-1}\right]=\mathbf{E}\left[X_{j, n}^{2} \mid \mathscr{F}_{n}^{j-1}\right]=\delta_{j, n}^{2}
$$

Покажем, что суммы $Z_{n}=\sum_{j=1}^{k_{n}} Z_{j, n}$ асимптотически нормальны. Для этого вероятностное пространство, на котором определены с.в. $\theta_{j, n}$, организуем следуюшим образом. Пусть $\xi$ - независимая от $X_{j, n}$ с.в., распределенная равномерно на $[0,1]$. Положим $\theta_{j, n}=1$ в том случае, если $j$-й знак после запятой в двоичном представлении $\xi$ есть 1, и $\theta_{j, n}=-1$ в противном случае. Из $\left(\mathrm{N}_{\mathrm{c}}\right)$ имеем:

$$
\mathbf{E}\left[\exp \left(i t Z_{n}\right) \mid \xi\right] \longrightarrow \exp \left(-\frac{t^{2}}{2}\right) \quad \text { при } n \rightarrow \infty
$$

при любом фиксированном значении $\xi$. Тогда по теореме Лебега о мажорируемой сходимости, так как $\left|\mathbf{E}\left[\exp \left(i t Z_{n}\right) \mid \xi\right]\right| \leqslant 1$,

$$
\mathbf{E} \exp \left(i t Z_{n}\right)=\mathbf{E}\left\{\mathbf{E}\left[\exp \left(i t Z_{n}\right) \mid \xi\right]\right\} \longrightarrow \exp \left(-\frac{t^{2}}{2}\right)
$$

Далее, так как по условию $\sum_{j} \delta_{j, n}^{2} \stackrel{\mathrm{p}}{\longrightarrow} 1$, для любого $M_{1}>1$

$$
\mathbf{P}\left\{\sum_{j} \delta_{j, n}^{2} \leqslant M_{1}\right\} \longrightarrow 1 \quad \text { при } n \rightarrow \infty \text {. }
$$


Так как $\max _{j} \delta_{j, n}^{2} \stackrel{\mathrm{p}}{\longrightarrow} 0$, для любого $\lambda>0$ при $n \rightarrow \infty$

$$
\mathbf{P}\left\{\max _{j} \delta_{j, n}^{2} \leqslant \lambda\right\} \rightarrow 1
$$

Следовательно, существует такая последовательность положительных чисел $\lambda_{n} \rightarrow 0$, что $\mathbf{P}\left\{\max _{j} \delta_{j, n}^{2} \leqslant \lambda_{n}\right\} \longrightarrow 1$ при $n \rightarrow \infty$.

Положим

$$
Y_{j, n}=Z_{j, n} I\left\{\delta_{j, n}^{2} \leqslant \lambda_{n}\right\} I\left\{\sum_{k=1}^{j} \delta_{k, n}^{2} \leqslant M_{1}\right\} .
$$

Поскольку величины $\delta_{1, n}^{2}, \ldots, \delta_{j, n}^{2}$ измеримы относительно $\sigma$-алгебры $\mathscr{K}_{n}^{j-1}$, условные распределения с.в. $Y_{j, n}$ остаются симметричными. Кроме того, поскольку

$$
\left\{\exists j: Y_{j, n} \neq Z_{j, n}\right\} \subseteq\left\{\sum_{j} \delta_{j, n}^{2}>M_{1}\right\} \bigcup\left\{\max _{j} \delta_{j, n}^{2}>\lambda_{n}\right\}
$$

имеем

$$
\mathbf{P}\left\{\exists j: Y_{j, n} \neq Z_{j, n}\right\} \leqslant \mathbf{P}\left\{\sum_{j} \delta_{j, n}^{2}>M_{1}\right\}+\mathbf{P}\left\{\max _{j} \delta_{j, n}^{2}>\lambda_{n}\right\} \longrightarrow 0
$$

при $n \rightarrow \infty$. Отсюда

$$
\mathbf{P}\left\{\sum_{j} Y_{j, n} \neq \sum_{j} Z_{j, n}\right\} \leqslant \mathbf{P}\left\{\exists j: Y_{j, n} \neq Z_{j, n}\right\} \longrightarrow 0 .
$$

Аналогично, полагая $\beta_{j, n}^{2}=\mathbf{E}\left(Y_{j, n}^{2} \mid \mathscr{K}_{n}^{j-1}\right)$, имеем

$$
\sum_{j=1}^{k_{n}} \beta_{j, n}^{2}=\sum_{j=1}^{k_{n}} I\left\{\delta_{j, n}^{2} \leqslant \lambda_{n}\right\} I\left\{\sum_{k=1}^{j} \delta_{k, n}^{2} \leqslant M_{1}\right\} \delta_{j, n}^{2}
$$

откуда

$$
\left\{\sum_{j} \beta_{j, n}^{2} \neq \sum_{j} \delta_{j, n}^{2}\right\} \subseteq\left\{\exists j: \delta_{j, n}^{2}>\lambda_{n}\right\} \cup\left\{\sum_{j} \delta_{j, n}^{2}>M_{1}\right\} .
$$

Следовательно,

$$
\mathbf{P}\left\{\sum_{j} \beta_{j, n}^{2} \neq \sum_{j} \delta_{j, n}^{2}\right\} \leqslant \mathbf{P}\left\{\sum_{j} \delta_{j, n}^{2}>M_{1}\right\}+\mathbf{P}\left\{\max _{j} \delta_{j, n}^{2}>\lambda_{n}\right\} \rightarrow 0 .
$$


Итак, для стохастической последовательности $\left\{Y_{j, n}, \mathscr{K}_{n}^{j}\right\}, 1 \leqslant$ $j \leqslant k_{n}$, выполнены условия утверждения А. Применяя его, получаем

$$
\sum_{j=1}^{k_{n}} \mathbf{E}\left\{Y_{j, n}^{2} I\left(\left|Y_{j, n}\right| \geqslant \varepsilon\right)\right\} \longrightarrow 0 \quad \text { при } n \rightarrow \infty .
$$

Однако

$$
\begin{aligned}
& \sum_{j=1}^{k_{n}} \mathrm{E}\left\{Y_{j, n}^{2} I\left[\left|Y_{j, n}\right| \geqslant \varepsilon\right] \mid \mathscr{K}_{n}^{j-1}\right\} \\
& =\sum_{j=1}^{k_{n}} I\left\{\delta_{j, n}^{2} \leqslant \lambda_{n}\right\} I\left\{\sum_{k=1}^{j} \delta_{k, n}^{2} \leqslant M_{1}\right\} \mathbf{E}\left\{Z_{j, n}^{2} I\left[\left|Z_{j, n}\right| \geqslant \varepsilon\right] \mid \mathscr{K}_{n}^{j-1}\right\} \\
& =\sum_{j=1}^{k_{n}} I\left\{\delta_{j, n}^{2} \leqslant \lambda_{n}\right\} I\left\{\sum_{k=1}^{j} \delta_{k, n}^{2} \leqslant M_{1}\right\} \mathbf{E}\left\{X_{j, n}^{2} I\left[\left|X_{j, n}\right| \geqslant \varepsilon\right] \mid \mathscr{F}_{n}^{j-1}\right\},
\end{aligned}
$$

и, аналогично сказанному выше, при $n \rightarrow \infty$

$$
\mathbf{P}\left\{\Lambda_{n}(\varepsilon) \neq \sum_{j} \mathbf{E}\left\{Y_{j, n}^{2} I\left(\left|Y_{j, n}\right| \geqslant \varepsilon\right) \mid \mathscr{K}_{n}^{j-1}\right\} \rightarrow 0 .\right.
$$

Из двух последних предельных соотношений получаем утверждение теоремы.

4. Доказательство теоремы 2. Как и при доказательстве предыдущей теоремы, достаточно доказать выполнение $(\mathrm{L})$ при $\sigma^{2}=1$ и условиях

$$
\max _{j \leqslant k_{n}} \delta_{j, n}^{2} \leqslant \lambda_{n}
$$

для некоторой последовательности $\lambda_{n} \rightarrow 0$ и

$$
\sum_{j=1}^{k_{n}} \delta_{j, n}^{2} \leqslant M_{1}
$$

для некоторой константы $M_{1}<\infty$.

Пусть $f_{j, n}(t)=\mathbf{E}\left\{\exp \left(i t X_{j, n}\right) \mid \mathscr{F}_{n}^{j-1}\right\}-$ условные характеристические функции с.в. $X_{j, n}, F_{j, n}\left(d x \mid \mathscr{F}_{n}^{j-1}\right)$ - соответствующие условные распределения.

Обозначим

$$
\mathscr{E}_{n}(t)=\prod_{j} f_{j, n}(t), \quad \mathscr{J}_{n}(t)=\exp \left\{\int_{-\infty}^{+\infty}\left(e^{i t x}-1-i t x\right) x^{-2} d K_{n}(x)\right\}
$$


где $K_{n}(u)=\sum_{j=1}^{k_{n}} \int_{-\infty}^{u} x^{2} F_{j, n}\left(d x \mid \mathscr{F}_{n}^{j-1}\right)-$ случайная функция, имеюшая в силу (16) ограниченную вариацию. ПІусть $K_{n}(d x)$ - соответствующая $K_{n}(u)$ случайная мера.

Зафиксируем произвольное $t$. Аналогично доказательству утверждения А предыдушего пункта имеем

$$
\begin{gathered}
|\ln | \mathscr{J}_{n}(t)|| \leqslant\left|\ln \mathscr{J}_{n}(t)\right| \leqslant M_{1} \frac{t^{2}}{2}, \\
\left|\ln \mathscr{E}_{n}(t)-\sum_{j=1}^{k_{n}}\left(f_{j, n}(t)-1\right)\right| \leqslant t^{4} \lambda_{n} M_{1} \longrightarrow 0, \\
\left|\sum_{j=1}^{k_{n}}\left(f_{j, n}(t)-1\right)\right|=\left|\sum_{j=1}^{k_{n}}\left(f_{j, n}(t)-1-\mathbf{E}\left(X_{j, n} \mid \mathscr{F}_{n}^{j-1}\right)\right)\right| \leqslant \frac{t^{2}}{2} M_{1} .
\end{gathered}
$$

Отскода

$$
|\ln | \mathscr{E}(t)|| \leqslant\left|\ln \mathscr{E}_{n}(t)\right| \leqslant\left(t^{4} \lambda_{n}+t^{2}\right) M_{1} .
$$

Следовательно, при каждом фиксированном $t$ сушествуют такие $c_{1}(t)$ и $c_{2}(t)$, что для всех $n$, начиная с некоторого номера (зависящего от $t$ ),

$$
\left|\mathscr{J}_{n}(t)\right|^{-1} \leqslant c_{1}(t), \quad\left|\mathscr{E}_{n}(t)\right|^{-1} \leqslant c_{2}(t) .
$$

Также аналогично доказательству утверждения $\mathrm{A}$

$$
\left|\mathscr{E}_{n}(t)-\mathscr{J}_{n}(t)\right| \stackrel{\mathrm{p}}{\longrightarrow} 0 \text { при } n \rightarrow \infty .
$$

Далее, поскольку в силу условия (N) с.в. $S_{n}$ асимптотически нормальна, существует последовательность усеченных с.в. $\widetilde{S}_{n}=S_{n} I\left\{\left|S_{n}\right| \leqslant\right.$ $\left.C_{n}\right\}, C_{n} \rightarrow \infty$, таких, что для некоторого $M_{2}<\infty$

$$
\mathbf{E}\left|\widetilde{S}_{n}\right|^{5} \leqslant M_{2} .
$$

Используя сделанные оценки, для каждого фиксированного $t$ имеем

$$
\begin{aligned}
& \left|\mathbf{E}\left(\mathscr{J}_{n}(t)\right)^{-1} \exp \left(i t \widetilde{S}_{n}\right)-\mathbf{E}\left(\mathscr{E}_{n}(t)\right)^{-1} \exp \left(i t S_{n}\right)\right| \\
& \quad \leqslant\left|\mathbf{E} \exp \left(i t \widetilde{S}_{n}\right)\left[\left(\mathscr{J}_{n}(t)\right)^{-1}-\left(\mathscr{E}_{n}(t)\right)^{-1}\right]\right| \\
& \quad+\left|\mathbf{E}\left(\mathscr{E}_{n}(t)\right)^{-1}\left[\exp \left(i t \widetilde{S}_{n}\right)-\exp \left(i t S_{n}\right)\right]\right| \\
& \left.\quad \leqslant c_{1}(t) c_{2}(t) \mathbf{E}\left|\mathscr{J}_{n}(t)-\mathscr{E}_{n}(t)\right|+c_{2}(t) \mathbf{E} \mid \exp \left(i t \widetilde{S}_{n}\right)-\exp \left(i t S_{n}\right)\right] \mid \longrightarrow 0 .
\end{aligned}
$$

Поскольку при каждом фиксированном $t$ для всех $n$, достаточно больших для того, чтобы выполнялось неравенство $\left|f_{j, n}(t)\right|>0$ для всех $j$, имеет 
место соотношение ([10]-[12], см. $[22$, с. 58$]) \mathbf{E}\left(\mathscr{E}_{n}(t)\right)^{-1} \exp \left(i t S_{n}\right)=1$, отсюда

$$
\mathrm{E}\left(\mathscr{J}_{n}(t)\right)^{-1} \exp \left(i t S_{n}\right) \longrightarrow 1 \text { при } n \rightarrow \infty .
$$

В силу (2) это соотношение можно переписать в виде

$$
\begin{aligned}
& \mathbf{E} \exp \left\{i t \widetilde{S}_{n}-\int_{-\infty}^{+\infty}\left(e^{i t x}-1-i t x+\frac{(t x)^{2}}{2}\right) x^{-2} d K_{n}(x)\right\} \\
& \longrightarrow \exp \left(\frac{-t^{2}}{2}\right) .
\end{aligned}
$$

Пусть $r=1,2,3, \ldots$. Обозначим

$$
\zeta_{r}=(r !)^{-1} \int_{-\infty}^{+\infty} x^{r} d \Phi(x), \quad \widehat{\zeta}_{r}=(r !)^{-1} \int_{-\infty}^{+\infty}|x|^{r} d \Phi(x),
$$

где $\Phi-$ стандартно нормальная функция распределения. Пусть

$$
k_{n}^{(r)}=\int_{-\infty}^{+\infty} x^{r} d K_{n}(x), \quad \widehat{k}_{n}^{(r)}=\int_{-\infty}^{+\infty}|x|^{r} d K_{n}(x) .
$$

Заметим, что

$$
k_{n}^{(r)}=\sum_{j=1}^{k_{n}} \mathrm{E}\left(X_{j, n}^{r+2} \mid \mathscr{F}_{n}^{j-1}\right), \quad \hat{k}_{n}^{(r)}=\sum_{j=1}^{k_{n}} \mathrm{E}\left(\left|X_{j, n}\right|^{r+2} \mid \mathscr{F}_{n}^{j-1}\right) .
$$

В силу (4) эти моменты при $r \leqslant 3$ существуют и их математические ожидания равномерно по $n$ ограничены.

Обозначим

$$
\alpha_{n}(t)=\int_{-\infty}^{+\infty}\left(e^{i t x}-1-i t x+\frac{(t x)^{2}}{2}\right) x^{-2} d K_{n}(x) .
$$

Пользуясь неравенством для остаточного члена разложения

$$
\left|e^{i t}-\sum_{k=0}^{m} \frac{(i t)^{k}}{k !}\right| \leqslant \frac{|t|^{m+1}}{(m+1) !},
$$

запишем для $m<5$ неравенство

$$
\left|\alpha_{n}(t)-\sum_{r=3}^{m}(i t)^{r} \frac{k_{n}^{(r-2)}}{r !}\right| \leqslant|t|^{m+1} \frac{\widehat{k}_{n}^{(m-1)}}{(m+1) !} .
$$

В силу неравенства $\left|e^{z}-1-z\right| \leqslant e^{|z|} z^{2} / 2$ и того, что $\left|\alpha_{n}(t)\right| \leqslant M_{1} t^{2}$, имеем для $|t| \leqslant 1$

$$
\begin{aligned}
2\left|\exp \left[-\alpha_{n}(t)\right]-1+\alpha_{n}(t)\right| & \leqslant \exp \left(M_{1} t^{2}\right)\left|\alpha_{n}(t)\right|^{2} \leqslant \exp \left(M_{1}\right)|t|^{6}\left(\hat{k}_{n}^{(1)}\right)^{2} \\
& \leqslant \exp \left(M_{1}\right)|t|^{6} k_{n}^{(2)}
\end{aligned}
$$


Тогда для $m \leqslant 4$

$$
\begin{aligned}
& \left|\mathbf{E} \exp \left[-\alpha_{n}(t)\right] \exp \left(i t \widetilde{S}_{n}\right)-\exp \left(-\frac{t^{2}}{2}\right)-\sum_{r=1}^{m}\left(\mu_{n r}-\zeta_{r}\right)(i t)^{r}\right| \\
& \quad \leqslant M_{3}|t|^{m+1}\left[\mathbf{E} \hat{k}_{n}^{(m-1)}+\hat{\zeta}_{m+1}\right]
\end{aligned}
$$

где $M_{3}$ - некоторое положительное число, $\mu_{n r}$ - коэффициенты тейлоровского разложения функции $\mathbf{E} \exp \left(-\alpha_{n}(t)\right) \exp \left(i t \widetilde{S}_{n}\right)$.

В силу (17) и (4) величины $\mathbf{E} \widehat{k}_{n}^{(m+1)}+\hat{\zeta}_{m+1}$, стояшие в правой части (19), при $m \leqslant 4$ ограничены равномерно по $n$. Покажем, что для $m \leqslant 4$

$$
\left|\mu_{n m}-\zeta_{m}\right| \longrightarrow 0 \text { при } n \rightarrow \infty \text {. }
$$

Сделаем это по индукции. Для $m=1, \mu_{n 1}=\mathbf{E} \widetilde{S}_{n} \longrightarrow \zeta_{1}=0$ в силу (17) и асимптотической нормальности $\widetilde{S}_{n}$. Предположим теперь, что $\left|\mu_{n r}-\zeta_{r}\right| \longrightarrow 0$ при $r<m$. Тогда из (19) при ненулевом $t$ имеем

$$
\begin{aligned}
& \mid\left[\mathbf{E} \exp \left[-\alpha_{n}(t)\right] \exp \left(i t \widetilde{S}_{n}\right)-\exp \left(-\frac{t^{2}}{2}\right)\right. \\
& \left.\quad-\sum_{r=1}^{m-1}\left(\mu_{n r}-\zeta_{r}\right)(i t)^{r}\right](i t)^{-m}-\left(\mu_{n m}-\zeta_{m}\right)\left|\leqslant M_{3}\right| t \mid\left[\mathbf{E} \widehat{k}_{n}^{(m-1)} \hat{\zeta}_{m+1}\right] .
\end{aligned}
$$

Предположим, что существует такое $\mu>0$, что $\left|\mu_{n m}-\zeta_{m}\right| \geqslant \mu$ для всех n. Согласно (4), при $r \leqslant 3$

$$
\mathbf{E} \widehat{k}_{n}^{(r)}=\mathbf{E} \int_{-\infty}^{+\infty}|x|^{r} d K_{n}(x) \leqslant \mathbf{E} \int_{-\infty}^{+\infty}\left(1+|x|^{3}\right) d K_{n}(x) \leqslant\left(1+M_{0}\right),
$$

поэтому можно выбрать $t$ так, чтобы

$$
M_{3}|t|\left[\mathbf{E} \hat{k}_{n}^{(m-1)}+\hat{\zeta}_{m+1}\right] \leqslant \frac{\mu}{4}
$$

равномерно по $n$. Тогда, согласно (18) и предположению индукции, при любом фиксированном $t \neq 0$ можно выбором $n$ сделать выражение в больших прямых скобках сколь угодно малым, в частности, меньшим $\mu / 4$, что противоречит сделанному предположению. Итак, $(20)$ доказано.

Согласно (20), при $n \rightarrow \infty$

$$
\mu_{n 4}-\zeta_{4}=(4 !)^{-1} \mathbf{E} \widetilde{S}_{n}^{4}-\zeta_{4}-(4 !)^{-1} \mathbf{E} k_{n}^{(2)}+(3 !)^{-1} \mathbf{E} \widetilde{S}_{n} k_{n}^{(1)} \longrightarrow 0 .
$$

Здесь в силу (17) и асимптотической стандартной нормальности $\widetilde{S}_{n}$

$$
(4 !)^{-1} \mathbf{E} \widetilde{S}_{n}^{4}-\zeta_{4} \longrightarrow 0
$$


Далее, условие (5) эквивалентно тому, что $\mathbf{E} S_{n} k_{n}^{(1)}=0$. В силу (4) и равномерной интегрируемости с.в. $S_{n}^{2}$, вытекающей из $(2),(16)$ и $(\mathrm{N})$, имеем

$$
\begin{aligned}
\mathbf{E} \widetilde{S}_{n} k_{n}^{(1)} & =\mathbf{E}\left\{\widetilde{S}_{n}-S_{n}\right\} k_{n}^{(1)}=\mathbf{E} S_{n} I\left(\left|S_{n}\right|>C_{n}\right) k_{n}^{(1)} \\
& \leqslant \sqrt{\mathbf{E}\left(S_{n}\right)^{2} I\left(\left|S_{n}\right|>C_{n}\right) \mathbf{E}\left(k_{n}^{(r)}\right)^{2}} \leqslant \sqrt{\mathbf{E}\left(S_{n}\right)^{2} I\left(\left|S_{n}\right|>C_{n}\right) \mathbf{E} k_{n}^{(2)}} \\
& \leqslant \sqrt{\left(1+M_{0}\right) \mathbf{E}\left(S_{n}\right)^{2} I\left(\left|S_{n}\right|>C_{n}\right)} \rightarrow 0 \text { при } n \rightarrow \infty .
\end{aligned}
$$

Таким образом, все члены в левой части (21), кроме $\mathbf{E} k_{n}^{(2)}$, стремятся к нулю при $n \rightarrow \infty$. Значит, из (21) имеем $\mathbf{E} k_{n}^{(2)} \rightarrow 0$, и, следовательно, $k_{n}^{(2)} \stackrel{p}{\longrightarrow} 0$.

В силу неравенства Чебышева при $n \rightarrow \infty$

$$
\sum_{j=1}^{k_{n}} \mathbf{E}\left\{X_{j, n}^{2} I\left(\left|X_{j, n}\right| \geqslant \varepsilon\right) \mid \mathscr{F}_{n}^{j-1}\right\}=\int_{|x| \geqslant \varepsilon} K_{n}(d x) \leqslant \varepsilon^{-2} k_{n}^{(2)} \stackrel{\mathrm{p}}{\longrightarrow} 0,
$$

т.е. получаем (L).

5. Доказательство следствия из теоремы 2. Пусть для с.в. $\left\{X_{j, n}\right\}$ выполнены условия следствия. Как показано выше, достаточно доказать выполнение (L) при условии (16). Рассмотрим последовательности с.в.

$$
Z_{j, n}=X_{j, n} I\left(\left|X_{j, n}\right| \leqslant 1\right)
$$

Поскольку

$$
\mathbf{P}\left\{\sum_{j=1}^{k_{n}} Z_{j, n} \neq \sum_{j=1}^{k_{n}} X_{j, n}\right\} \leqslant \sum_{j=1}^{k_{n}} \mathbf{P}\left\{Z_{j, n} \neq X_{j, n}\right\} \leqslant \mathbf{E} \Lambda(1) \longrightarrow 0,
$$

суммы $\sum_{j=1}^{k_{n}} Z_{j, n}$ асимптотически нормальны. Отсюда, из (2), (3), (5') и (16) следует выполнение для серий с.в. $\left\{Z_{j, n}\right\}$ всех условий теоремы 2 , кроме условия мартингальности (1). Так как

$$
\sum_{j=1}^{k_{n}}\left|\mathbf{E}\left\{X_{j, n} I\left(\left|X_{j, n}\right| \leqslant 1\right) \mid \mathscr{F}_{n}^{j-1}\right\}\right| \leqslant \Lambda(1)
$$

нетрудно убедиться, что здесь применимо доказательство теоремы 2 (с очевидными изменениями), и для серий $\left\{Z_{j, n}\right\}$ выполнено условие Линдеберга, т.е.

$$
\sum_{j=1}^{k_{n}} \mathbf{E}\left\{X_{j, n}^{2} I\left[1 \geqslant\left|X_{j, n}\right| \geqslant \varepsilon\right] \mid \mathscr{F}_{n}^{j-1}\right\} \stackrel{\mathrm{p}}{\longrightarrow} 0
$$

Так как по условию следствия $\Lambda(1) \stackrel{\mathrm{p}}{\longrightarrow} 0$, отскода получаем $(\mathrm{L})$. 


\section{СПИСОК ЛИТЕРАТУРЫ}

1. Brown B., Eagleson G. Martingale convergence to infinitely divisible laws with finite variance. - Trans. Amer. Math. Soc., 1971, v. 162, p. 449-453.

2. Brown B. Martingale central limit theorems. - Ann. Math. Statist., 1971, v. 42, p. 59-66.

3. Вектиель А. Д. Курс теории случайных процессов, 2-е изд. М.: Наука, 1996, $400 \mathrm{c}$.

4. Ganssler $P$., Hausler $E$. Remarks on the functional central limit theorem for martingales. - Z. Wahrscheinlichkeitstheor. verw. Geb., 1979, v. 50, p. 237-243.

5. Dvoretzky A. Asymptotic normality for sums of dependent random variables. - Proceedings of the 6th Berkeley Symposium on Mathematical Statistics and Probability, v. 3, Berkeley: Univ. of California Press, 1972, p. 513-535.

6. Жакод Ж., ПІиряев $A . H$. Предельные теоремы для случайных процессов, т. 1 , 2. M.: Наyкa, 1994.

7. Kabanov Yu., Liptser R., Shiryayev A. Some limit theorems for simple point processes (martingale approach). - Stochastics, 1980, v. 3, p. 203-216.

8. Колмогоров А. Н., Фомия C. В. Элементы теории функций и функционального анализа, 6-е изд. М.: Наука, 1989, 636 с.

9. Lindvall $T$. Weak convergence of probability measures and random functions in the function space $D[0, \infty)$. - J. Appl. Probab., 1973, v. 10, p. 109-121.

10. Лuпчер $P$. III., ШІиряев $A$. H. Функциональная центральная предельная теорема для семимартингалов. - Теория вероятн. и ее примен., 1980, т. XXV, в. 4, с. 683703.

11. Липчер P. III., ШІиряев $A$. H. О необходимых и достаточных условиях в функциональной центральной предельной теореме для семимартингалов. - Теория вероятн. и ее примен., 1981, т. XXVI, в. 1, с. 132-137.

12. Липчер P. III., IIиряев A. Н. Теория мартингалов. М.: Наука, 1986, 512 с.

13. Лозв $M$. Теория вероятностей. М.: ИЛ, 1962, 720 с.

14. McLeish $D$. L. Invariance principles for dependent variables. - Wahrscheinlichkeitstheor. verw. Geb., 1975, v. 32, p. 165-178.

15. Прохоров $Ю$. . В. Сходимость случайных процессов и предельные теоремы теории вероятностей. - Теория вероятн. и ее примен., 1956, т. I, в. 2, с. 117-238.

16. Rebolledo $R$. Central limit theorems for local martingales. - Wahrscheinlichkeitstheor. verw. Geb., 1980, v. 51, p. 269-286.

17. Rootzen $H$. On the functional central limit theorem for martingales, II. - Wahrscheinlichkeitstheor. verw. Geb., 1980, v. 51, p. 79-93.

18. Скороход $A$. B. Об одном представлени и случайных величин. - Теория вероятн. и ее примен., 1976, т. XXI, в. 3, с. 645-648.

19. Scott $D$. J. Central limit theorems for martingales and for processes with stationary increments using Skorokhod representation theorem. - Adv. Appl. Probab., 1975, v. 5, p. 119-137.

20. Stone $C$. Weak convergence of stochastic processes defined on a semifinite time interval. - Proc. Amer. Math. Soc., 1963, v. 14, p. 694-696.

21. Hall D., Heyde J. Martingale Central Limit Theorem and its Applications. New York: Academic Press, 1980.

22. IIIияев $A$. Н. Вероятность, 2-е изд. М.: Наука, 1988, 640 с.

Поступила в редакцию

6.III.1997 